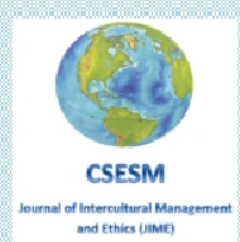

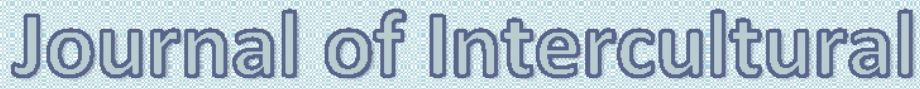

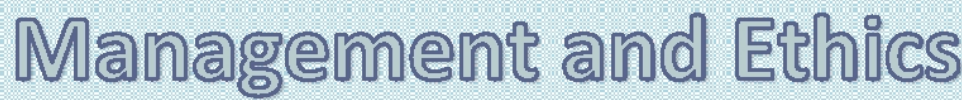

\author{
IOME
}

ISSN 2601 - 5749, ISSN-L 2601 - 5749

\section{published by zy \\ Center for Socio-Economic Studies and Multiculturalism \\ lasi, Romania \\ Waw csesmorg}




\section{Special Editor}

\section{Professor Beatrice Gabriela Ioan, PhD, MD}

Grigore T.Popa University of Medicine and Pharmacy of Iasi, Romania

E-mail: ioanbml@yahoo.com

\section{TABLE OF CONTENT}

Editorial

Beatrice Gabriela Ioan

Global Pandemics: Considerations of Public Health, Human Rights, and Bioethics

SanaLoue

Reactions of Bioethics to the Covid-19 Pandemic 19

Elena Toader, Luiza Palamaru, Tudor Stefan Rotaru, Oana Hrisca Eva

Implications of Cognitive-Behavioural Therapy in Changing Illness Representations .27

Ancuța Elena Păduraru, Camelia Soponaru

Healthcare Workers - Heroes and Villains in Covid-19 Pandemic. The Ethical

Communication

Ioana Silistraru

Ethical Dilemmas in the Therapeutic Management of Patients with Sars-Cov-2 Infection .. 45 Claudia Elena Pleşca, Ioana Hunea, Maria Obreja, Oana Stămăteanu, Delia Luchian, Irina Dima, Larisa Miftode, Tudorița Gabriela Părângă, Egidia Miftode, Simona Apostu, Camelia Bucur, Daniela Leca

Covid - 19 through the Window of Age 55

Elena Toader, Daniela Damir, Tudor Winzinger

Ethical Aspects of "Do Not Resuscitate" Orders in the Context of the Covid-19 Pandemic 61 Iulius Connor Gramma, Gema Bacoanu, Beatrice Gabriela Ioan

Ethics of the Human-Animal Relationship in the Covid-19 Pandemic .67

Cristin Coman, Diana Ancuta 


\title{
IMPLICATIONS OF COGNITIVE-BEHAVIOURAL THERAPY IN CHANGING ILLNESS REPRESENTATIONS
}

\author{
Ancuța Elena Păduraru ${ }^{1}$, Camelia Soponaru ${ }^{1}$ \\ „„Alexandru Ioan Cuza” University, Iasi, Romania \\ *corresponding author, e-mail: ancuta.bojian@yahoo.com
}

\begin{abstract}
Objectives: Due to the obvious links between disease representations and health outcomes, the interest of researchers is to develop interventions aimed to correct dysfunctional beliefs about the disease, in order to improve the condition of patients. Cognitive behavioural therapy (CBT) is a proper theoretical basis for the formulation of such programs. Our goal was to conduct a theoretical review - in order to identify the state of knowledge on the implementation and effectiveness of such programs - by highlighting their main features.

Methods: In order to conduct our review, we searched for articles in 5 databases "Proquest", "Reaxys", "Sage", "Science Direct" and "Springer" - and we have analysed the studies that have implemented or intend to implement interventions for restructuring illness representations, based on cognitive behavioural therapy.

Results: We analysed 44 articles, most of which are protocols of future studies. However, the existing results are promising. In general, such interventions comprise 4 to 10 sessions that can be provided in various settings - individually, group-based, or mixed aiming to form accurate illness representations among patients by providing information through direct interaction between the specialist and the patient or through the written material.

Conclusions: The CBT interventions may determine positive modifications of symptoms and coping strategies in patients, with beneficial effects on the evolution of health. Though they are still in the early stages, research findings are promising and - beyond any doubt - cognitive-behavioural therapy is worthy of attention from researchers who investigate patients' illness representations.
\end{abstract}

Keywords: illness representation, cognitive-behavioural interventions, common-sense model

\section{Introduction}

The authors Chater and Loewenstei (Chater \& Loewenstei, 2016) argue that people have an inner drive to confer meaning upon their daily experiences, their life and the world in general. Therefore, once people start experiencing certain changes in health state - i.e., symptoms - or receive the diagnosis of a condition, they try to make sense of them, by formulating a common-sense hypothesis (Asnani, Barton-Gooden, Grindley, \& KnightMadden, 2017). More precisely, based on the available information, persons will generate cognitive and emotional representations of their illness (Wiener, Cassisi, Paulson, Husson, \& Gupta, 2017). This concept demonstrates its importance in the field of health through the findings within the scientific literature, which has recorded a rapid increase. The rising importance highlights the existence of significant relations between the representations of patients and the subsequent outcomes of illness - emotional distress, rehabilitation and disability, adherence to treatment and survival (Christensen, Frostholm, Ørnbøl, \& Schröder, 2015). In other words, patients' behaviour is in close correlation with the beliefs they have about their illness (Hagger \& Orbell 2003). If patients have negative beliefs about the 
consequences of illness and if their perceived level of symptoms is low, they may end up using maladaptive coping mechanisms, such as denial and avoidance, thus their emotional state acquiring negative valences (Hallas, Wray, Andreou, \& Banner 2011; Morgan, VilliersTuthnill, Barker, \& McGee, 2014). Due to the relations between illness representation and the evolution of the patients' state, the researchers' interest shifted towards the development of interventions leading to the corrections of the dysfunctional beliefs about illness, in order to improve the patients' outcomes (Petrie \& Weinman, 2012).

Cognitive-behavioural therapy may be a proper approach to modifying negative perceptions about illness, given that - besides restructuring the distorted information of patients about illness - it may favour the emergence of more positive and functional cognitions, helping patients acquire more confidence in their control capacity concerning illness management (Riskind, 2006; Morgan et al., 2014).

In order to conduct our review, we searched for articles by the following keywords: "cognitive-behavioural therapy and illness beliefs", "cognitive intervention and illness perception", "cognitive-behavioural therapy and illness representations", "cognitivebehavioural therapy and illness perceptions", "modify illness perceptions" in 5 databases, i.e., "Proquest", "Reaxys", "Sage", "Science Direct" and "Springer". We excluded articles that approached mental illness and non-chronic diseases; we focused only on those using the principles of cognitive-behavioural therapy to improve illness representations. The abstracts of 310 articles were analysed, 44 of which were read in order to identify the main features of intervention programs designed to restructure dysfunctional illness representations, starting from cognitive-behavioural therapy. The most important ideas are presented below.

\section{Illness representations}

Leventhal and his colleagues have studied for several years the way people represent and then react to the stressors threatening their health. The authors theorising eventually theorised the common-sense model. Hence, they found evidence attesting that people - in their attempt to understand and regulate health-related behaviour - develop implicit illness theories (Kemp, 1996). This assumption shares the same standpoint with the one by Lazarus and Folkman, according to which people's experiences and views are determined partially by the objective situation they experience and partially by what people bring along in that situation (Carver \& Vargas, 2011). Thus, illness perception begins with the onset of somatic experiences, while the extent to which somatic experiences are evaluated as threatening is influenced by a person's previous experience with illness, as well as by the information received from their social and cultural setting (Chapman et al., 2015). According to Leventhal et al., (Leventhal, Meyer, \& Nerenz, 1980, p. 10; Heijmans, de Ridder, \& Bensing, 1999; McManus, 2011) illness representations are the personal, implicit, common-sense beliefs of an individual about illness, a subjective viewpoint of individuals concerning their illness comprising two fields: cognitive representation and emotional representation.

Illness representations are constructed based on the information coming from three sources. The first source of information is represented by the "laic" information derived from the previous social conversations or from the cultural knowledge and the influences of illness. The second source of information is outlined by the significant persons for the individual, as well as by authority figures, such as specialist physicians, nurses, etc. The third source of information is obtained from the previous experiences of the individual in relation to illness, as well as from the present experiences of illness (Hart, 2006).

Illness representations include information on the identity of illness, assumptions in what concerns the underlying causes, the timeline, consequences and perceived controllability or curability (Carver \& Vargas, 2011). Identity unites under one label the symptoms experienced by persons, whereas in order to determine the timeline of illness, we 
use temporal measurement units: it may be acute, chronic or cyclical. The dimension of causes comprises identifications and descriptions of the stimuli considered to be responsible for the onset of illness, while the consequences refer to the implications determined by illness on a short term, but also on a long term in the most important fields of life: social, professional, familial, etc. Not least, the fifth dimension of illness representation controllability/curability - contains beliefs and feelings related to the capacity to control or to cure that a person and/or treatment may exercise on illness (Marin, 2010; van Erp et al., 2017). Besides these dimensions - encountered at the cognitive level of illness representation - the common-sense model also underlines the importance of the emotional level, comprising the emotions persons experience in relation to illness. The coping strategies implemented by a person will be selected depending on the contents at both the cognitive level of illness representations and the emotional level (Diefenbach \& Leventhal, 1996). Each of the two levels has three components: a) cognitive/emotional representation, b) coping strategies and c) evaluation (Leventhal et al., 1980).

The information that persons come across are assimilated in a continuous feedback loop for them to be evaluated and subsequently to have an impact on the coping mechanisms adopted (Kotsis, Voulgari, Tsifetaki, Drosos, Carvalho, \& Hyphantis, 2014). As persons acquire new information about illness, based on their own experience with illness (the emergence of a new symptom) or from the environment (from health professionals, friends, family, culture), illness representations continue developing and restructuring (Asnani et al., 2017). These modifications further influence the coping strategies used by patients to face illness, as well as the evaluation of their effectiveness (Hart, 2006).

As soon as the modifications of illness perceptions are intentional - using scientifically-based information - within interventions that are part of illness treatment, beneficial effects on health (Petrie \& Weinman, 2012; Jayanti, Foden, Wearden, \& Mitra, 2016; Norton et al., 2014), quality of life and treatment adherence (Van der Have et al., 2013; van Erp et al., 2017; Ashur, Shan, Bosseri, Morisky, \& Shamsuddin, 2015) can be recorded. For instance, Petrie, Perry, Broadbent, and Weinman (2011) have applied an intervention with the purpose of stimulating adherence to the treatment preventing the onset of asthma crises among young adults. The initial phase involved the evaluation of patients' illness and treatment representations; subsequently, throughout the following weeks, they received messages meant to modify dysfunctional beliefs. Findings have highlighted significant changes at the level of illness perceptions and adherence to treatment; these changes persisted up to six months after the intervention.

In addition, the more accurate illness representations, the more functional relationship between the physician and the patient, while understanding information about illness becomes easier (Broadbent et al., 2009).

Illness representations are not important only throughout a person's disease, but also after healing. Wiener and his colleagues (2017) have demonstrated that informational support - in what concerns illness, medical tests and treatment - is associated with less threatening illness representations, in their turn associated with less distress. The relationship between informational support and distress was mediated by illness representations (Wiener, Cassisi, Paulson, Husson, \& Gupta, 2017). Several types of interventions have proven to be useful on illness perceptions. However, in order to increase their clinical efficiency, a series of improvements - i.e., shorter intervention period, the possibility of online delivery and lower costs - must be implemented (Jones, Ellis, Nash, Stanfield, \& Broadbent, 2016). Cognitivebehavioural therapy is already based on these principles and it serves as reference for psychotherapeutic interventions (Pinto et al., 2017). 


\section{Cognitive-behavioural therapy}

Cognitive-behavioural therapy connects the way people think with their behavioural choices and with the emotion they experience (Cook et al., 2015). In case of patients diagnosed with a certain illness, cognitive-behavioural therapy has the main purpose of increasing the quality of life by identifying and intervening on the factors maintaining symptomatology, by developing beneficial behaviours for health and by regulating the symptoms (Selinheimo et al., 2016). One of the ways to attain the goals is to identify the dysfunctional representations about illness and to restructure them in order to make them realistic (Scheenen et al., 2017). The dysfunctional illness representations of a patient were formulated based on the previous experiences and they influence the present actions. Therefore, we can create - through cognitive-behavioural therapy - new learning experiences by providing accurate information, in order to correct the irrational beliefs and to determine adaptive behaviours subsequently (Talebi, Tabatabaye, \& Vahedi, 2018). Furthermore, these new learning situations generated by CBT provide the possibility of pointing out new healthy alternatives for the patient and new coping procedures (Beauregard, 2014; Scheenen et al., 2017).

Psychoeducation provided in CBT encourages the patients to adopt an active role in illness management and treatment, by enriching the knowledge on pain management and by reducing dysfunctional beliefs on medication, which eventually reduces the intensity of the symptoms, such as cancer-related pain (Ogbeide \& Fitch-Martin, 2016). Cognitivebehavioural therapy is a proper approach for the intervention on illness perception, because it increases people's understanding of the relationship between body and mind and of the way in which this connection influences the evolution of illness, thus integrating information from the biological, psychological and social sphere (Pinto et al., 2017; De Gucht, 2015). This type of therapy improves perceived control of illness management, increases coherence about illness and it allows a more accurate identification of illness consequences (van Erp et al., 2017; Doerr, Jopp, Chajewski, \& Naster, 2017; Scheenen et al., 2017); more precisely, it has effects on the contents of illness representation dimensions. By approaching the dysfunctional ruminations about illness, we may obtain significant progresses in treating depression in case of patients with certain chronic conditions (Julien, Rimes, \& Brown., 2016).

Cognitive-behavioural therapy has proven effective in reducing the symptoms in case of somatic diseases such as cancer (Watson, White, Lynch, \& Mohammed, 2017) and of emotional conditions, such as anxiety disorders (Hofmann \& Smits, 2008), as well as illnessrelated stress. The following step would involve providing online therapy; the clinical and financial efficacy of which could be determined within comparative studies, where we would assess the outcomes of cognitive-behavioural therapy offered by a therapist, compared to those of online therapy (Everitt et al., 2015; Hudson et al., 2016). The advantages of online CBT - besides the improvement of psychological functioning - would be the elimination of logistic barriers, as well as lower dropout rates (Douma, Scholten, Maurice-Stam, \& Grootenhuis., 2018; Kallestad et al., 2018).

Behavioural therapy - derived from the CBT - has been used successfully in the reduction of the frequency and intensity of headaches among teenagers and adults; the patients eventually acquire higher perceived control of the symptoms (Faedda et al., 2016; Day, Thorn, \& Rubin, 2014). Huang - along with his colleagues - (2016) has underpinned that the intervention based on cognitive-behavioural therapy helps diabetic patients adapt psychologically to illness and increase their glycaemic control, thus improving the persons' capacity of controlling their diabetes and of obtaining healthier outcomes (Huang et al., 2016).

Within cognitive-behavioural therapy, patients come first, while intervention at a cognitive, emotional and behavioural level concerns not only the attainment of health, but 
also the adherence to a healthier lifestyle (Zhang et al., 2016). Furthermore, the strong point of this approach is the lack of adverse effects (Faedda et al., 2016).

\section{Cognitive-behavioural therapy as an intervention method on illness representations}

Research on the implications of cognitive-behavioural therapy on illness perceptions is still in the early stages; mostly protocols of future studies have been published. Corbett and his collaborators $(2016 ; 2017)$ have published such a protocol, through which they propose an online intervention program aiming to identify the effects of cognitive-behavioural therapy on fatigue perception and illness self-management, in case of cancer-surviving patients. The assumption of the authors cited above argues that the intervention of CBT may determine beneficial modifications of the symptoms and coping strategies used by cancer survivors (Corbett et al., 2017). Picariello and his collaborators (2018) have envisaged testing similar assumptions, but their protocol will be applied to haemodialyzed patients.

The mechanisms through which cognitive-behavioural therapy acts upon the perceptions of patients concerning their symptoms or the treatment they follow may be provided and explained by the common-sense model (Corbett et al., 2017). The emergence of these research protocols is based on the findings of various studies, proving that - unlike the concomitant provision of cognitive-behavioural therapy - it leads to the formulation of more positive illness perceptions, with beneficial effects on the evolution of health (Chilcot \& Moss-Morris, 2013; Cope et al., 2017).

Howard and Dupont (2014) have used the principles of the common-sense model and the cognitive-behavioural techniques in order to develop a notebook/ manual meant to be used by patients diagnosed with chronic obstructive pulmonary disease, in order to manage the panic states and the choking sensations. Compared to the patients receiving the regular informative leaflets, the patients who used the intervention manual recorded significant improvements at the level of self-healthcare and of emotional and health status (Howard and Dupont, 2014). Correcting the beliefs of patients on illness identity and on treatment controllability may lead to the reduction of reported somatic symptoms (Hyphantis et al., 2014), which may be attained by providing the patients with accurate information about the illness (Husson et al., 2013). The alteration of illness representations influences health-related outcomes (Bijsterbosch et al., 2009), potentially through cognitive-behavioural therapy (Chapman et al., 2015). Intervention on illness perceptions - equivalent with determining a more coherent understanding of one's illness - is associated with increased quality of life among patients (Janssen et al., 2013), while negative perceptions are related to higher mortality risk (Thong et al., 2016; Parfeni, Nistor, \& Covic, 2013). It is important for health specialists to focus on evaluating the patients' illness perceptions, because the significance ascribed may know different variations from the scientific point of reference. The need to be cured may even be overshadowed by the need to understand and form a coherent representation of the illness (Shukla \& Pandey, 2013). The need to know and understand one's illness has also been highlighted by Løchting and his collaborators (2016), who have demonstrated within their study that educational intervention based on cognitive therapy has determined a faster improvement of illness perceptions among patients. Moreover, the patients who have been provided with the intervention by psychotherapists have recorded better results compared to the group of patients for whom intervention was carried out by the generalist physicians (Løchting et al., 2016).

Scientific literature has underscored the need to carry out studies researching the efficiency of interventions meant to alter illness representations (Parfeni et al., 2013; De Gucht, 2015; Ashur et al., 2015; Doerr et al., 2017), and such studies have already been published. 
On a general note, the clinical trials with the purpose of intervening on patients' illness perceptions, on symptoms and effects - through cognitive-behavioural therapy - are based on the following principles:

- 4 to 10 intervention sessions are provided;

- intervention is individual, group-based, or mixed;

- generally, the objectives of the intervention programs are as follows: providing scientific information about illness; identifying negative or inaccurate thoughts about illness and restructuring them; developing coping procedures for the symptoms of illness and increasing self-control level in relation to disease management.

- intervention may take the form of written material (e.g., leaflets) given by specialists by direct interaction with the patient or online (Scheenen et al., 2017; Pinto et al., 2017; Sandhu et al., 2016; Selinheimo et al., 2016; Howard \& Dupont, 2014; Picariello et al., 2018).

Christensen and his colleagues (2015) have demonstrated within their randomised controlled trial that cognitive-behavioural therapy may be used successfully to determine modifications at the level of illness representations for patients with functional somatic syndrome, and these changes have mediated partially certain improvements at the level of physical health, even one year after the treatment. The most important contribution in the mediating effect was increased confidence in a person's control capacity concerning illness. Another conclusion - with clinical implications - of the afore-cited authors is that the improvement of patients' illness representations (concerning the dimensions of controllability and consequences, as well as emotional representations) may be a key-element in obtaining successful treatment (Christensen et al, 2015). This conclusion is also supported by the findings of the study conducted by Chilcot and Moss-Morris (2013), illustrating that cognitive-behavioural therapy entailed positive changes at the level of patients' illness representations in case of irritable bowel syndrome. More precisely, cognitive-behavioural therapy has allowed patients to understand their illness more coherently, to have fewer perceptions about the serious and painful consequences of illness, thus increasing their perceived control on illness (Chilcot \& Moss-Morris, 2013). Knoop and his collaborators (2011) have used cognitive-behavioural therapy in order to test whether its potential impact on fatigue representations and on perceived fatigue level, in case of patients with multiple sclerosis. Following the intervention, the change of negative fatigue representations had a significant role in reducing fatigue. In other words, increasing fatigue comprehension, perceived control exercised by the person on fatigue, developing beliefs according to which illness has less serious consequences on a physical and emotional level leads to a reduction in fatigue gravity (Knoop, van Kessel, \& Moss-Morris, 2011).

Though they are still in the early stages research findings are promising and - beyond any doubt - cognitive-behavioural therapy is still worthy of attention from researchers investigating patients' illness representations and their adherence to treatment.

\section{Conclusions}

The communication between physician and patient is very important, while the evaluation of patients' illness representations may provide us with the opportunity of identifying the level of inconsistency between the way they understand illness and the way they relate to treatment (the two sides of this relationship). The improvement of communication between the two sides, the increased empathy degree and the language adjusted to patients may lead to a more accurate understanding of illness by the patient. Naturally, these strategies may not suffice, given that people construct illness representations not only based on information received from the specialist physician. An important role is 
played by their own previous experiences with illness, by non-scientific information from friends and family, by the cultural and religious beliefs specific to a person's environment. The essence of this model is that people suffering from a certain illness use common sense to elaborate illness representations, starting from their own knowledge and experiences. In order to correct distorted illness representations - that may be assimilated to hypotheses about illness - cognitive-behavioural therapy has proven particularly effective.

Illness representations have two main levels: cognitive and emotional. The contents of the two levels have a significant impact on the coping mechanisms used by a person to face illness, which determines important effects on the evolution of illness and on health-related outcomes. Therefore, in order to intervene on the restructuring of this construct, a theoretical foundation is necessary. Such foundation is meant to provide the proper tools for identifying and understanding this subtle dynamic between cognition, emotion, coping strategies and outcome. These needs are very much consistent with the principles of cognitive-behavioural therapy. Other advantages of such type of intervention include short duration, applicability to a wide array of conditions and the possibility of providing it online.

\section{Acknowledgement}

This work was co-funded by the European Social Fund, through Operational Programme Human Capital 2014-2020, project number POCU/380/6/13/123623, project title < $<$ PhD Students and Postdoctoral Researchers Prepared for the Labour Market!>>

\section{References}

Ashur, S. T., Shan, S. A., Bosseri, S., Morisky, D. E., \& Shamsuddin, K. (2015). Illness perceptions of Libyans with T2DM and their influence on medication adherence: a study in a diabetes center in Tripoli. Libyan Journal of Medicine, 10, 29797.

Asnani, M. R., Barton-Gooden, A., Grindley, M., \& Knight-Madden, J. (2017) Disease Knowledge, Illness Perceptions, and Quality of Life in Adolescents with Sickle Cell Disease: Is There a Link?. Global Pediatric Health, 4, 1-10.

Beauregard, M. (2014). Functional neuroimaging studies of the effects of psychotherapy. Dialogues Clin Neurosci. 16(1), 75-81.

Bijsterbosch, J., Scharloo, M., Visser, A.W., Watt, I.,Meulenbelt, I. Huizinga, T.W., et al. (2009). Illness perceptions in patients with osteoarthritis: changeover time and association with disability. Arthritis and rheumatism, 61,1054-61.

Broadbent, E., Ellis, C.J., Thomas, J., Gamble, G., \& Petrie, K.J. (2009). Further development of an illness perception intervention for myocardial infarction patients: A randomized controlled trial. Journal of Psychosomatic Research, 67(1), 17-23.

Carver, S. C., \& Vargas, S. (2011). Stress, Coping, and Health In Froedman, H. S. (Ed.) The Oxford Handbook of Health Psychology. Oxford University Press: New York.

Chapman, S.C.E., Barnes, N., Barnes, M., et al. (2015) Changing adherence-related beliefs about ICS maintenance treatment for asthma: feasibility study of an intervention delivered by asthma nurse specialists. BMJ Open; 5:e07354.

Chater, N., \& Loewenstei, G. (2016). The under-appreciated drive for sense-making. Journal of Economic Behavior \& Organization. 126, Part B, 137-154.

Chilcot, J., \& Moss-Morris, R. (2013). Changes in illness-related cognitions rather than distress mediate improvements in irritable bowel syndrome (IBS) symptoms and disability following a brief cognitive behavioural therapy intervention. Behaviour research and therapy, 51(10), 690-5.

Christensen, S. S., Frostholm, L., Ørnbøl, E., \& Schröder, A. (2015). Changes in illness perceptions mediated the effect of cognitive behavioural therapy in severe functional somatic syndromes. Journal of Psychosomatic Research, 78, 363-370. 
Cook, S.A., Salmon, P., Dunn, G., Holcombe, C., Cornford, P., \& Fisher, P. (2015). A Prospective Study of the Association of Metacognitive Beliefs and Processes with Persistent Emotional Distress after Diagnosis of Cancer. Cognitive Therapy and Research 39, 51-60.

Cope, S. R., Smith, J. G., King, T., \& Agrawal, N. (2017). Evaluation of a pilot innovative cognitive-behavioral therapy-based psychoeducation group treatment for functional non-epileptic attacks. Epilepsy \& Behavior, 70, 238-244.

Corbett, T., Walsh, J.C., Groarke, A., Moss-Morris, R., Morrissey, E., \& McGuire, B.E. (2017). Cancer-Related Fatigue in Post-Treatment Cancer Survivors: Theory-Based Development of a Web-Based Intervention. JMIR Cancer,3(2), e8.

Corbett, T., Walsh, J.C., Groarke, A.M., Morrissey, E., \& McGuire, B.E. (2016) Protocol for a pilot randomised controlled trial of an online intervention for post-treatment cancer survivors with persistent fatigue. BMJ Open 2016; 6:e011485.

Day, M. A., Thorn, B. E., \& Rubin, N. J. (2014) Mindfulness-based cognitive therapy for the treatment of headache pain: A mixed-methods analysis comparing treatment responders and treatment non-responders. Complementary Therapies in Medicine, 22, $278-285$.

De Gucht, V. (2015). Illness perceptions mediate the relationship between bowel symptom severity and health-related quality of life in IBS patients. Quality of Life Research, 24, $1845-1856$.

Diefenbach, M. A., \& Leventhal, H. (1996). The Common-Sense Model of Illness Representation: Theoretical and Practical Considerations, Journal of Social Distress and the Homeless, 5( L).

Doerr, J. M., Jopp, D. S., Chajewski, \& Naster, U. M. (2017). Patterns of control beliefs in chronic fatigue syndrome: results of a population-based survey. BMC Psychology, $5(6)$.

Douma, M., Scholten, L., Maurice-Stam, H., \& Grootenhuis, M. A. (2018). Online cognitivebehavioral based group interventions for adolescents with chronic illness and parents: study protocol of two multicenter randomized controlled trials. BMC Pediatrics, 18, 235.

Everitt, H., Landau, S., Little, P., Bishop, F. L., McCrone, P. et al. (2015) Assessing Cognitive behavioural Therapy in Irritable Bowel (ACTIB): protocol for a randomised controlled trial of clinical-effectiveness and cost-effectiveness of therapist delivered cognitive behavioural therapy and web based self-management in irritable bowel syndrome in adults. BMJ Open; 5:e008622.

Faedda, N., Cerutti, R., Verdencchia, P., Migliorini, D., Arruda, M., \& Guidetti, V (2016). Behavioral management of headache in children and adolescents. The Journal of Headache and Pain, 17, 80.

Hagger, M.S., \& Orbell, S. (2003). A meta-analytic review of the common-sense model of illness representations. Psychology \& Health, 18(2), 141-184.

Hallas, C.N., Wray, J., Andreou, P., \& Banner, N.R. (2011). Depression and perceptions about heart failure predict quality of life in patients with advanced heart failure. Heart \& Lung: The Journal of Acute and Critical Care, 40(2), 111-121.

Hart, P. L., (2006). Illness representation, emotional distress, coping strategies, and coping efficacy as predictors of patient outcomes in type 2 diabetes, Dissertation, Georgia State University, http://scholarworks.gsu.edu/nursing_diss/2

Heijmans, M., M., de Ridder, D., \& Bensing, J.(1999). Dissimilarity in patients' and spouses' representations of chronic illness: Exploration of relations to patient adaptation. Psychology \& Health, 14(3), 451 - 466 
Hofmann, S.G., \& Smits, J.A. (2008) Cognitive-behavioral therapy for adult anxiety disorders: a meta-analysis of randomized placebo-controlled trials. Journal of Clinical Psychiatry, 69, 621-32.

Howard, C., \& Dupont, S. (2014). 'The COPD breathlessness manual': a randomised controlled trial to test a cognitive-behavioural manual versus information booklets on health service use, mood and health status, in patients with chronic obstructive pulmonary disease. NPJ primary care respiratory medicine, 24, 14076. doi.org/10.1038/npjpcrm.2014.76

Huang, C-Y., Lai, H-L., Chen, C-I., Lu, Y-C., Li, S-C., et al. (2016). Effects of motivational enhancement therapy plus cognitive behaviour therapy on depressive symptoms and health-related quality of life in adults with type II diabetes mellitus: a randomised controlled trial. Quality of Life Research, 25, 1275-1283.

Hudson, J.L., Moss- Morris, R., Game, D., et al. (2016). Improving distress in dialysis (iDiD): a feasibility two-arm parallel randomised controlled trial of an online cognitive behavioural therapy intervention with and without therapist-led telephone support for psychological distress in patients undergoing haemodialysis. BMJ Open; 6:e011286.

Husson, O., Thong, M.S., Mols, F., Oerlemans, S., Kaptein, A.A., \& vandePoll- Franse, L.V. (2013). Illness perceptions in cancer survivors: what is the role of information provision? Psycho-Oncology, 22, 490-8. doi.org/10.1002/pon.3042

Hyphantis, T., Kroenke, K., Papatheodorou, E., Paika, V., Theochpoulos, N., Ninou, A., Tomenson, B. et al. (2014). Validity of the Greek version of the PHQ15-item Somatic Symptom Severity Scale in patients with chronic medical conditions and correlations with emergency department use and illness perceptions. Comprehensive Psychiatry, $55,1950-1959$.

Janssen, V., De Gucht, V., van Exel, H., \& Maes, S. (2013). Changes in Illness Perceptions and Quality of Life During Participation in Cardiac Rehabilitation. International Journal of Behavioral Medicine, 20, 582-589

Jayanti, A., Foden, P., Wearden, A., \& Mitra, S. (2016). Illness Beliefs in End Stage Renal Disease and Associations with Self-Care Modality Choice. PLoS ONE 11(7), e0154299.

Jones, A. S., Ellis, C. J., Nash, M., Stanfield, B., \& Broadbent, E. (2016). Using Animation to Improve Recovery from Acute Coronary Syndrome: A Randomized Trial. Annals of Behavioral Medicine, 50, 108-118.

Julien, C. L., Rimes, K. A., \& Brown, R. G. (2016). Rumination and behavioural factors in Parkinson's disease depression. Journal of Psychosomatic Research, 82, 48-53.

Kallestad, H., Vedaa, Ø., Scott, J., Morken, G., Pallesen, S. et al. (2018) Overcoming insomnia: protocol for a large-scale randomised controlled trial of online cognitive behaviour therapy for insomnia compared with online patient education about sleep. BMJ Open 2018, 8:e25152.

Kemp, S. (1996). The role of illness representations in the process of coping and psychosocial adjustment among adults with epilepsy. [PhD thesis, University of Leeds].

Knoop, H., van Kessel, K., \& Moss-Morris, R. (2011). Which cognitions and behaviours mediate the positive effect of cognitive behavioural therapy on fatigue in patients with multiple sclerosis? Psychological Medicine, 42(01), 205-213.

Kotsis, K., Voulgari, P. V., Tsifetaki, N., Drosos, A. A., Carvalho, A. F., \& Hyphantis, T. (2014) Illness perceptions and psychological distress associated with physical health-related quality of life in primary Sjögren's syndrome compared to systemic 
lupus erythematosus and rheumatoid arthritis. Rheumatology International, 34, 16711681.

Leventhal, H., Diefenbach, M., \& Leventhal, E. A. (1992). Illness Cognition: Using Common Sense to Understand Treatment Adherence and Affect Cognition Interactions, Cognitive Therapy and Research, 16(2), 143-163.

Leventhal, H., Meyer, D. \& Nerenz, D. (1980). The common-sense model of illness danger. In: Rachman, S. (Ed.), Medical psychology, 2, 7-30. Pergamon, New York.

Løchtinga, I., Storheima, K., Wernerd, E.L., Småstuen Cvancarovaa, M., \& Grotlea, M. (2016). Evaluation of individualized quality of life and illness perceptions in low back pain. A patient education cluster randomized controlled trial. Patient Education and Counseling, 99, 1992-1998.

Marin, A. M. (2010). Concepts of illness among children of different ethnicities, socioeconomic backgrounds, and genders. [M.Appl.Psy. thesis, San Diego State University]

McManus, M. S. (2011). Illness representation and medication adherence of patients with chronic kidney disease. [PhD thesis, Indiana University]

Morgan, K., Villiers-Tuthnill, A., Barker, M., \& McGee, H. (2014). The contribution of illness perception to psychological distress in heart failure patients. BMC Psychology, 2(50).

Norton, S., Hughes, L. D., Chilcot, J., Sacker, A., van Os, S., Toung, A., \& Done, J. (2014). Negative and positive illness representations of rheumatoid arthritis: a latent profile analysis. Journal of Behavioral Medicine, 37, 524-532.

Ogbeide, S., \& Fitch-Martin, A. (2016). Cancer Pain Management: Implications for Psychologists. Psychology, Community \& Health, 5(1), 61-79.

Parfeni, M., Nistor, I., \& Covic, A. (2013) A systematic review regarding the association of illness perception and survival among end-stage renal disease patients. Nephrology Dialysis Transplantation, 28(10), 2407-14.

Petrie, K.J., \& Weinman, J. (2012). Patients' perceptions of their illness: the dynamo of volition in health care. Current Directions in Psychological Science, 21, 60-5.

Petrie, K. J., Perry, K., Broadbent, E., \& Weinman, J. (2011). A text message program designed to modify patients' illness and treatment beliefs improves self-reported adherence to asthma preventer medication. British Journal of Health Psychology. Advance online publication.

Picariello,F., Moss-Morris, R., Macdougall, I. C., Norton, S., \& Da Silva-Gane, M. (2018) Cognitive-behavioural therapy (CBT) for renal fatigue (BReF): a feasibility randomised-controlled trial of CBT for the management of fatigue in haemodialysis (HD) patients. BMJ Open; 8:e20842.

Pinto, P.R., Paredes, A.C., Costa, P., et al. (2017) Effectiveness of two psychological interventions for pain management, emotional regulation and promotion of quality of life among adult Portuguese men with haemophilia (PSY HaEMOPEQ): study protocol for a single centre prospective randomised controlled trial. BMJ Open; 7:e016973.

Riskind, J.H. (2006). Links Between cognitive-behavioral hope-building and positive psychology: applications to a psychotic patient. Journal of Cognitive Psychotherapy, 20(2), 171-182.

Sandhu, H., Bernstein, C.J., Davies, G., et al. (2016) Combined cognitive- behavioural and mindfulness programme for people living with dystonia: a proof-of concept study. BMJ Open; 6:e011495.

Scheenen, M. E., Visser-Keizer, A. C., de Koning, M. E., van der Horn, H. J., van de Sande, P., van Kessel, M., ... Spikman, J. M. (2017). Cognitive Behavioral Intervention 
Compared to Telephone Counseling Early after Mild Traumatic Brain Injury: A Randomized Trial. Journal of Neurotrauma, 34(19), 2713-2720.

Selinheimo, S., Vuokko, A., Sainio, M., et al. (2016). Comparing cognitive behavioural psychotherapy and psychoeducation for non-specific symptoms associated with indoor air: a randomised control trial protocol. BMJ Open; 6: e011003.

Shukla, A., \& Pandey, P. (2013). Cognitive reframing strategies to cope with spousal chronic illness. Indian Journal of Health and Wellbeing, 4(9), 1660-1664.

Talebi, M., Tabatabaye, K. R., \& Vahedi, E. (2018). Application of Cognitive-Behavioral Therapy in Obstructive Sleep Apnea: Comparison of Combined Treatment, and CPAP Therapy on Executive Functions of the Brain. Sleep and Hypnosis, 20(3), 148-159.

Thong, M. S. Y., Kaptein, A. A., Vissers, P. A. J., Vreugdenhil, G., \& van de Poll-Franse, V. (2016). Illness perceptions are associated with mortality among 1552 colorectal cancer survivors: a study from the population-based PROFILES registry. Journal of Cancer Survivorship, 10, 898-905.

Van der Have, M., Minderhoud, I.M., Kaptein, A.A., Leenders, M., Siersema, P.D., Fidder, H.H., Oldenburg, B. (2013). Substantial impact of illness perceptions on quality of life in patients with Crohn's disease. Journal of Crohn's and Colitis, 7, 292-301.

van Erp, S. J. H, Brakenhoff, L. K. M. P., Vollmann, M.,van der Heijde, D., Veenendaal, R.A., Fidder, H. H., Hommes, D. W. et al. (2017). Illness Perceptions and Outcomes in Patients with Inflammatory Bowel Disease: Is Coping a Mediator? International Journal of Behavioral Medicine, 24, 205-214.

Watson, M., White, C., Lynch, A., \& Mohammed, K (2017). Telephone-delivered individual cognitive behavioural therapy for cancer patients: An equivalence randomised trial. Psycho-Oncology, 26, 301-308.

Wiener, C. H., Cassisi, J. E., Paulson, D., Husson, O., \& Gupta, R. A. (2017). Information support, illness perceptions, and distress in survivors of differentiated thyroid cancer. Journal of Health Psychology, 1-9.

Zhang, Y., Mei, S., Yang, R., Chen, L., Gao, H., \& Li, L. (2016). Effects of lifestyle intervention using patient-centered cognitive behavioral therapy among patients with cardiometabolic syndrome: a randomized, controlled trial. BMC Cardiovascular Disorders, 16(227). 